\title{
Application of an Innovate Energy Balance to Investigate Viscoelastic Problems
}

\author{
Saeed Shahsavari ${ }^{1 *}$, Mehran Moradi² \\ ${ }^{1}$ Department of Mechanical Engineering, Isfahan University of Technology \\ Isfahan 84156-83111, Iran. \\ Email: s.shahsavari [AT] me.iut.ac.ir \\ 2 Department of Mechanical Engineering, Isfahan University of Technology \\ Isfahan 84156-83111, Iran. \\ Email: moradi [AT] iut.ac.ir
}

\begin{abstract}
Modeling and investigating of energy distribution especially the wasted one is very important in viscoelastic problems. In this article, an applied energy model based on separation of energy components of the system is extracted and expanded to apply in linear viscoelastic problems, although this method is applicable in nonlinear problems as well. It is assumed that the whole energy of the system can be divided into two parts: Residual and noninertial energies. The non-inertial energy is the sum of the energies that do not depend on the inertia of the system, while residual energy is the remaining of total energy. When an amount of energy is applied to the system, by determining the non-inertial energy from a novel energy conservation equation, the residual energy can be calculated. Some basic viscoelastic examples are investigated and obtained results will be compared with the expected ones.
\end{abstract}

Keywords - Energy Balance; Residual Energy; Non-inertial energy; Viscoelasticity

\section{INTRODUCTION}

Energy dissipation in solids can be occurred by several different mechanisms, and although ultimately these all result in the mechanical energy being transformed into heat, two main dissipative processes are involved. The first ty pe is known as 'static hysteresis'; that means the energy loss per cycle is independent from frequency, where the main cause may be ass ociated simply with the 'static' non-linear stress-strain behavior of the materials. Another type is known as 'viscosity' properties of the body, according to which many materials show losses that are related to the velocity gradients set up by the vibrations. The forces producing these losses may be considered to have a viscous nature and mention that the mechanical behavior will depend upon the rate of strain. This is sue is the subject of the 'linear viscoelasticity' [1].

When some energy is applied to a viscoelastic body, a part of the applied energy is dissipated as heat because of viscosity properties of matter, but the remaining is stored as reversible energy than is known as potential energy. It is frequently of interest to determine, for a viscoelasticity body at a given mode of displacement or deformation, the whole energy of deformation as well as the amount of energy stored or dissipated that can be investigated by studying the structure of vis cose material[2,3,4].

The total deformational energy can be stated as follows:

$W(t)=W_{s}(t)+W_{d}(t)$

Where $W_{s}(t)$ is the energy stored and $W_{d}(t)$ is the energy dissipated. Equation(1) can be rewritten as follows:

$\dot{W}(t)=\dot{W}_{s}(t)+\dot{W}_{d}(t)$

Where all terms will refer to the unit volume of the body. Equation (2) may be looked upon as the definition of a viscoelastic material [4]. Based on this definition, a viscoelastic material is one in which the totalapplied energy is partly stored elastically and remaining dissipated as heat. Since applied energy to the body can only be stored ordis sipated, Eq (1) is quite generally true and also all terms are positive. What makes it a viscoelastic relation is the interpretation of the stored energy as purely potential energy. The precise form of Eq. (1) depends, of course, on the nature of the material on the one hand, and on the mode of the displacementor deformation on the other. It should be noted that equation(1) is not limited to investigating the linear behavior of viscous matters. The behavior studied here does not depend on the assumption that the response can be modeled by springs and dashpots as mechanical model. It may be simply supposed of energy storing and dissipating mechanisms without recognizing them with mechanical models, and modify the proof as needed as 
an energy studying. For energy studying, it is necessary to know enough information on the applied deformation and the rate of the applied energy $[5,6,7,8]$.

Reversible stored energy is known as elas tically or potential energy. Energy may also be stored inertially, called here as residual energy, which may be encountered in fast loading experiments such as response to impulsive excitation, or in wave propagation at high frequency. Although in the linear theory of viscoelasticity, inertial energy storage plays no role in investigating the behavior of the matter, one may wish to calculate it [4]. The deformation of these structures requires exchange of residual energy and various internal elastic energies. This energy exchange is realized through special coupling of the transport of the internal elastic variables and the induced elastic stress which can be determined by using of the Hamilton's principle $[9,10]$. Some researchers have studied the structure and energy dis sipation by using of other methods $[11,12,13]$. Therefore, general analy sis of equation (1) and residual energy of body as well as energy dissipated or s tored will be frequently of interest.

In this paper, by presenting a novel glance to energy components and their balance, the residual energy is defined and calculated as well as non-inertial energy which is the sumof the all energies that do not depend on theinertia of the system. Als o, the presented approach is expanded to different basic linear vis coelasticity models and the results are discussed.

\section{MATHEMATICAL MODELING}

The certain effects of vis coelastic material behavior manifest themselves in a time-dependent response to loading and accompanying energy dissipation. The res ponse of these materials will depend on the amount and rate of applied energy to the body. In fact, it can be stated that the relaxation or creep occurs when a vis coelastic matter is exposed to quasi-static displacement or loads and their changes. These phenomena are treated typically in the time and in the frequency domain, respectively [14].

By examining the activated energy components in the performed process, the non-inertial energy can be investigated, and thus, by applying the principle of energy conservation, the inertia-dependent energies of the body, hereafter called residual energy, are calculated. The presented approach can provide a practical idea for analy zing the relevant problems .

It is assumed that the total energy of the systemcan be stated as follows:

$U_{T}=U_{V}+U_{R}$

Where $U_{T}$ is total energy, $U_{V}$ is non-inertial energy and $U_{R}$ is residual energy.

From the pers pective of non-inertial energies, when an amount of energy is applied to the system, some of the energy components will be activated. Between these activated components, some of themchange independently and changing of other components will be dependent on the independent components. In this paper the bellow equation is used for noninertial energies:

$U_{V}=U_{V_{s}}+U_{V_{d}}$

$U_{V_{S}}=\left(u_{1}+u_{2}+\cdots+u_{m}\right)+\left[g_{1}+\cdots+g_{k}\right]$

$U_{V_{d}}=\left[h_{1}+\cdots+h_{n}\right]$

Where:

$g_{j}=g_{j}\left(u_{1}, u_{2}, \ldots, u_{m}\right)$

$h_{p}=h_{p}\left(\dot{u}_{1}, \ldots, \dot{u}_{m}\right)$

Where $U_{V_{S}}$ is a part of non-inertial energy that is stored and $U_{V_{d}}$ is the remaining that is dis sipated. Equation (4) has the main idea of equation (1) which describes the performed process based on energy components in viscoelastic bodies. In equation (5), the functions of $g_{j}$ show the dependent components as a function of independent components while the functions of $h_{p}$ show the effects of applying energy rate on the structure of non-inertial energy of body. In a vis coelastic body, functions of $g_{j}$ are activated because of the poisson's ratio of material while functions of $h_{p}$ are activated because of the vis cosity properties.

The variation of equation (4):

$\delta U_{V}=\sum_{i=1}^{m} \delta u_{i}+\sum_{j=1}^{k} \sum_{i=1}^{m} \frac{\partial g_{j}}{\partial u_{i}} \delta u_{i}+\sum_{p=1}^{n} \sum_{i=1}^{m} \frac{\partial h_{p}}{\partial \dot{u}_{i}} \delta \dot{u}_{i}$

The eq. (9) means that an amount of energy variation $\delta U_{T}$ is given to the body and the variation of energy components due to this process are considered, so as a result the following changes will be occur in the independent components:

$\delta u_{1}=\alpha_{1} \delta U_{T}$

: 
$\delta u_{m}=\alpha_{m} \delta U_{T}$

The coefficients $\alpha_{\mathrm{i}}$ as loading coefficients will be depended on how energy is applied to the body as well as materials properties of the body, therefore:

$\delta \dot{u}_{i}=\dot{\alpha}_{i} \delta U_{T}+\alpha_{i} \delta \dot{U}_{T}$

By assuming that:

$\alpha_{s}=\left[\sum_{i=1}^{m} \alpha_{i}\left(1+\sum_{j=1}^{k} \frac{\partial g_{j}}{\partial u_{i}}\right)\right]$

$\alpha_{d}=\left[\sum_{i=1}^{m} \dot{\alpha}_{i}\left(\sum_{p=1}^{n} \frac{\partial h_{p}}{\partial \dot{u}_{i}}\right)\right]$

$\alpha=\alpha_{s}+\alpha_{d}$

$\beta=\sum_{i=1}^{m} \alpha_{i}\left(\sum_{p=1}^{n}\left(\frac{\partial h_{p}}{\partial \dot{u}_{i}}\right)\right)$

The variation of non-inertial energy can be rewritten as follows:

$\delta U_{V}=\alpha \delta U_{T}+\beta \delta \dot{U}_{T}$

And also:

$\delta U_{V_{S}}=\alpha_{S} \delta U_{T}$

$\delta U_{V_{d}}=\alpha_{d} \delta U_{T}+\beta \delta \dot{U}_{T}$

By using equation (3), the variation of the residual energy in a performed process can be written as follows:

$\delta U_{R}=\delta U_{T}-\delta U_{V}$

By placing the equation (16) in (19):

$\delta U_{R}=(1-\alpha) \delta U_{T}-\beta \delta \dot{U}_{T}$

Equation (20) gives the residual energy of the body as a function of the amount and rate of applied energy to the body as well as coefficients $\alpha$ and $\beta$.

\section{LINEAR VISCOELASTICITY}

In this part, using the presented approach, some basic linear vis coelasticity models are investigated and obtained results will be compared with the expected ones. At first, a vis coelastic body under uniformuniaxial stress, as shown in fig ure 1, is considered. This example can show the basis of presented approach clearly.

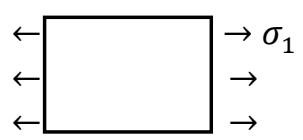

Figure 1: Viscoelastic body under uniformuniaxial stress

Because of uniaxial stress, Pois son's ratio does not have an effect on the non-inertial energy in this problem, so there is no need to use functions $g_{j}$. If we consider the effects of vis cosity as a dependent component and als o use a linear model, then the following statement can be used to express non-inertialenergy:

$U_{V}=u_{e}+c_{1} \dot{u}_{e}+c_{2}$

Where $u_{e}$ is the elastic energy as independent component and $h_{e}=c_{1} \dot{u}_{e}$ is used to consider the effect of linear viscosity of body as dependent component. Also $c_{1}$ and $c_{2}$ are constant, where $c_{1}$ depends on the material's viscosity and elastic material properties and $c_{2}$ depends on the initial conditions.

By using of equations (14) and (15):

$\alpha=\alpha_{e}+c_{1} \dot{\alpha}_{e}$

$\beta=c_{1} \alpha_{e}$

Which $\alpha_{e}$ represents the proportion of energy that is converted to elastic energy. Therefore equation (16) can be rewritten as follows:

$\delta U_{V}=\left(\alpha_{e}+c_{1} \dot{\alpha}_{e}\right) \delta U_{T}+\left(c_{1} \alpha_{e}\right) \delta \dot{U}_{T}$ 
By defining how energy is applied to body, the variation of non-inertialenergy can be calculated. The dis sipated and stored parts of the non-inertial energy are also as follows:

$\delta U_{V_{S}}=\left(\alpha_{e}\right) \delta U_{T}$

$\delta U_{V_{d}}=\left(c_{1} \dot{\alpha}_{e}\right) \delta U_{T}+\left(c_{1} \alpha_{e}\right) \delta \dot{U}_{T}$

Equations (25) and (26) state that the stored part depends on the amount of applied energy only while the dissipated part depends on the amount as well as rate of the applied energy. This is in line with the definition of viscoelastic materials. Equation (26) also takes a clear description to the dissipated energy and its reasons. Figure 2 shows linear viscoelastic kelvin model.

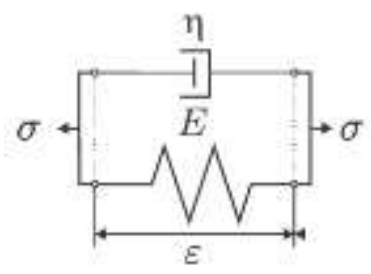

Figure 2: Kelvin-Voight model

For elements shown in figure 2 based on the parameters defined in equation (21):

$\frac{\partial u_{e}}{\partial \varepsilon}=E \varepsilon$
$\frac{\partial u_{d}}{\partial \varepsilon}=\eta \dot{\varepsilon}$

That $\mathrm{u}_{\mathrm{e}}$ and $\mathrm{u}_{\mathrm{d}}$ are stored and dissipated energies respectively, therefore:

$\frac{\partial^{2} u_{e}}{\partial \varepsilon \partial t}=\frac{E}{\eta} \frac{\partial u_{d}}{\partial \varepsilon}$

$u_{d}=\frac{\eta}{E} \dot{u}_{e}+f(t)$

Where $f(t)$ is a function of time related to initial condition. To determine $\alpha_{e}$ as loading coefficient for viscoelastic kelvin model, it can be written based on energy conservation principle and equations (21) and (30):

$\delta U_{T}=\delta u_{e}+\delta u_{d}$

Where:

$\delta u_{d}=c_{1} \delta \dot{u}_{e}=\frac{\eta}{E} \delta \dot{u}_{e}$

$\delta u_{d}=\left(\frac{\mathrm{n}}{E}\right)\left[\dot{\alpha}_{e} \delta U_{T}+\alpha_{e} \delta \dot{U}_{T}\right]$

Therefore:

$\delta U_{T}=\left(\alpha_{e}\right) \delta U_{T}+\left(\frac{\mathrm{n}}{E}\right)\left[\dot{\alpha}_{e} \delta U_{T}+\alpha_{e} \delta \dot{U}_{T}\right]$

$\dot{U}_{T}=\left(\alpha_{e}\right) \dot{U}_{T}+\left(\frac{\mathrm{n}}{E}\right)\left[\dot{\alpha}_{e} \dot{U}_{T}+\alpha_{e} \ddot{U}_{T}\right]$

That yield:

$\dot{\alpha}_{e}+\left[\frac{\dot{U}_{T^{+}}\left(\frac{\eta}{E}\right) \ddot{U}_{T}}{\left(\frac{\eta}{E}\right) \dot{U}_{T}}\right] \alpha_{e}=\left(\frac{E}{\eta}\right)$

Therefore, $\alpha_{e}$ can be calculated from equation (36) which strongly depends on how energy would be applied to the system.

As another way, if dis sipated energy is considered as independent component:

$U_{V}=u_{d}+c_{1} \dot{u}_{d}+c_{2}$

Therefore:

$\alpha=\alpha_{d}+c_{1} \dot{\alpha}_{d}$

$\beta=c_{1} \alpha_{d}$

$\delta U_{V}=\left(\alpha_{d}+c_{1} \dot{\alpha}_{d}\right) \delta U_{T}+\left(c_{1} \alpha_{d}\right) \delta \dot{U}_{T}$ 
This results in:

$\delta U_{V_{S}}=\left(\alpha_{d}\right) \delta U_{T}$

$\delta U_{V_{d}}=\left(c_{1} \dot{\alpha}_{d}\right) \delta U_{T}+\left(c_{1} \alpha_{d}\right) \delta \dot{U}_{T}$

Equation (37) expres ses the energy structure of Maxwell vis coelastic model, as shown in figure 3. For this model:

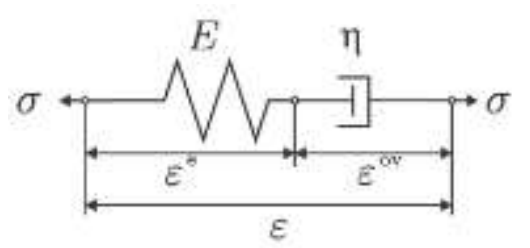

Figure 3: Viscoelas tic Maxwell model

$\frac{\partial u_{d}}{\partial \varepsilon^{O V}}=\eta \dot{\varepsilon}^{O V}=E \varepsilon^{e}=\frac{\partial u_{e}}{\partial \varepsilon^{e}}$

By supposing that second derivative of $\varepsilon^{O V}$ with respect to time is zero, this equation yields:

$u_{e}=\frac{\eta}{2 E} \dot{u}_{d}$

Therefore, for this model, the loading coefficient $\alpha_{d}$ can be obtained as bellows:

$\dot{\alpha}_{d}+\left[\frac{\dot{U}_{T^{+}}\left(\frac{\eta}{2 E}\right) \ddot{U}_{T}}{\left(\frac{\eta}{2 E}\right) \dot{U}_{T}}\right] \alpha_{d}=\left(\frac{2 E}{\eta}\right)$

As seen the general formof non-inertial energy structure of Kelvin and Maxwell models are similar.

This procedure could be generalized for an arbitrary kelvin elements connected in series as shown in figure 4 . The noninertial energy structure is :

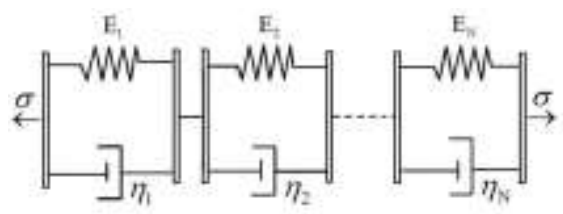

Figure 4: Generalized kelvin elements

$U_{V}=u_{e 1}+u_{e 2}+\cdots+u_{e N}+c_{e 1} \dot{u}_{e 1}+c_{e 2} \dot{u}_{e 2}+\cdots+c_{e N} \dot{u}_{e N}+$ constant

This is also true forgeneralized Maxwell elements, as shown in figure 5:

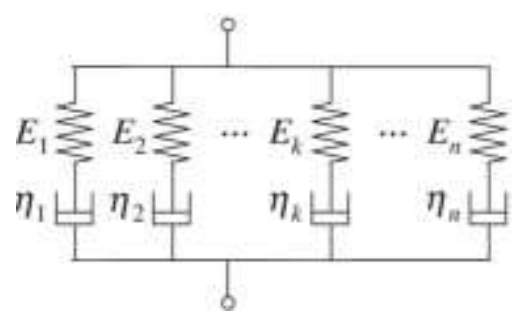

Figure 5: Generalized Maxwell element

$U_{V}=u_{d 1}+u_{d 2}+\cdots+u_{d N}+c_{d 1} \dot{u}_{d 1}+c_{d 2} \dot{u}_{d 2}+\cdots+c_{d N} \dot{u}_{d N}+$ constant

Indeed obtained non-inertial energy equations show that when an amount of energy is applied to the system, what would be the feasible changes of the systembased on the way the energy is applied to it.

\section{CONCLUSIONS}

Based on the way considering independent and dependent energy components, an energy distribution to the system could be pres ented to consider the physical properties as well as feasible directions. 
The whole energy of the body is divided into two parts: residual energy and non-inertial energy. The non-inertial energy equation depends on the physical properties of the body and its variation is defined based on the amount and rate of applied energy to the systemas well as the loading coefficients.

By considering energy conservation principle, the non-inertial energy as well as residual energy could be calculated. The presented approach applied to linear vis coelastic models which resulted in obtaining a first order differential equation to calculate dissipated or storing energy based on the way that the whole energy is applied to the system, in other words energy transfer rate and acceleration. For Kelvin and Maxwell models, similar general form of non -inertialenerg y equation was obtained.

\section{REFERENCES}

[1] Caputo, Michele, and Francesco Mainardi. "A new dissipation model based on memory mechanism." Pureand Applied Geophysics 91.1 (1971): 134-147.

[2] Del Piero, Gianpietro, and Luca Deseri. "On the concepts of state and free energy in linear viscoelasticity." Archive for Rational Mechanics and Analysis 138.1 (1997): 1-35.

[3] Colinas-Armijo, Natalia, Mario Di Paola, and Francesco P. Pinnola. "Fractional characteristic times and dis sipated energy in fractional linear viscoelasticity." Communications in Nonlinear Science and Numerical Simulation 37 (2016): 14-30.

[4] Bland, David Russell. The theoryoflinear viscoelasticity. Courier Dover Publications, 2016.

[5] Hongbin, Yang, et al. "Energy dissipation behaviors of a dispersed viscoelastic microsphere system." Colloids and Surfaces A: Physicochemical and Engineering Aspects 487 (2015): 240-245.

[6] Fabrizio, Mauro, and Angelo Morro. Mathematical problems in linear viscoelasticity. Vol. 12. Siam, 1992.

[7] Deseri, Luca, Giorgio Gentili, and Murrough Golden. "An explicit formula for the minimum free energy in linear viscoelasticity." Journal of elasticity 54.2(1999): 141-185.

[8] Tschoegl, Nicholas W., Wolfgang G. Knauss, and Igor Emri. "Pois son's ratio in linear viscoelasticity-a critical review." Mechanics ofTime-Dependent Materials 6.1 (2002): 3-51.

[9] Lei, Zhen, Chun Liu, and Yi Zhou. "Global existence for a 2D incompressible viscoelastic model with small strain." Communications in Mathematical Sciences 5.3(2007): 595-616.

「10] Ghayesh, Mergen H. "Dynamics of functionally graded viscoelastic microbeams." International Journal of Engineering Science 124 (2018): 115-131.

[11] Likhtman, Alexei E., Sathish K. Sukumaran, and Jorge Ramirez. "Linear viscoelasticity from molecular dynamics simulation of entangled polymers." Macromolecules 40.18 (2007): 6748-6757.

[12] Gentili, Giorgio. "Maximum recoverable work, minimum free energy and state space in linear viscoelasticity." QuarterlyofApplied Mathematics 60.1 (2002): 153-182.

[13] Colby, Ralph H. "Structure and linear viscoelasticity of flexible polymer solutions: comparis on of polyelectrolyte and neutral polymer solutions." Rheologica Acta 49.5 (2010): 425-442.

[14] Pettermann, Heinz E., and Antonio DeSimone. "An anisotropic linear thermo-viscoelastic constitutive law." Mechanics oftime-dependent materials 22.4(2018): 421-433. 